\title{
JOINT CONTINUITY OF MEASURABLE BIADDITIVE MAPPINGS
}

\author{
JENS PETER REUS CHRISTENSEN AND PAL FISCHER
}

(Communicated by Richard R. Goldberg)

\begin{abstract}
The main result of this paper is the following theorem. If $G_{1}, G_{2}$ and $G_{3}$ are abelian Polish groups and $C: G_{1} \times G_{2} \rightarrow G_{3}$ is a Christensen measurable biadditive mapping, then $C$ is jointly continuous.
\end{abstract}

1. Introduction. Biadditive mappings have been discussed many times in the literature, often in conjunction with the parallelogram law $[\mathbf{1 0}, \mathbf{8}, \mathbf{5}, \mathbf{7}]$, etc. An important problem in the theory of biadditive mappings is to find conditions which ensure the continuity of the mappings.

There are many results about continuity of measurable homomorphisms in quite general settings but in the case of biadditive mappings we are aware only of studies of measurable solutions of the associated parallelogram law in a general (Banach space) setting. [9]

In this work it will be assumed that the domain of the biadditive mappings is the product of two abelian Polish (or more general) groups and the range space is again an abelian Polish (or more general) group. Hence, in general, there is no associated parallelogram law with the aid of the diagonal of the domain; thus only the structure of the group can be used.

We wil discuss separately continuous and measurable biadditive mappings, where both universal and Christensen measurability will be considered. We refer to [2] for the definition of the Haar zero sets in an abelian Polish group and to [6 and 4] for the notion of Christensen measurability. Since there are no Fubini types of theorem for the small sets we consider [2], a quite intricate construction has to be given to prove our main result. Nevertheless, we believe that these results show the power of these small sets.

Throughout this paper we shall use the following notations. If $G_{i}$ is an abelian Polish group, then $O_{G_{i}}$ is the neutral element of $G_{i}$, and $d_{i}$ is a complete translation invariant metric, which is compatible with the topology of $G_{i}$.

2. Universally measurable mappings. Our first result shows that a universally measurable biadditive mapping is jointly continuous in an abelian Polish group setting.

Received by the editors March 31, 1987.

1980 Mathematics Subject Classification (1985 Revision). Primary 43A46; Secondary 39B70, $22 \mathrm{~A} 10$.

Key words and phrases. Joint continuity, universal measurability, Christensen measurability, biadditive mappings.

Work supported in part by the NSERC of Canada under grant A-8421. 
THEOREM 1. Let $G_{1}, G_{2}$ and $G_{3}$ be abelian Polish groups and let $C: G_{1} \times G_{2} \rightarrow$ $G_{3}$ be a universally measurable biadditive mapping. Then $C$ is jointly continuous.

ProOF. Let $g_{1}$ be a fixed element of $G_{1}$, then $C\left(g_{1}, \cdot\right): G_{2} \rightarrow G_{3}$ is a group homomorphism. In an obvious way, $G_{2}$ can be identified with the closed subset $\left\{g_{1}\right\} \times G_{2}$ of $G_{1} \times G_{2}$ (the mapping $g_{2} \mapsto g_{1} \times g_{2}$ is a homomorphism). Now, using the fact that a closed set is also universally measurable, and that the restriction of a universally measurable mapping to a closed subset is relatively universally measurable, we can conclude that $C\left(g_{1}, \cdot\right)$ is a universally measurable group homomorphism. Therefore $C\left(g_{1}, \cdot\right)$ is continuous [3, p. 114]. In an entirely similar way, we can show that $C\left(\cdot, g_{2}\right): G_{1} \rightarrow G_{3}$ is a continuous group homomorphism for every fixed $g_{2} \in G_{2}$. Hence $C: G_{1} \times G_{2} \rightarrow G_{3}$ is a separately continuous mapping. With the aid of a result of Calbrix and Troallic [1, Corollary 3, p. 648] we can conclude that there exists at least one $g_{1} \in G_{1}$ and at least one $h_{\infty} \in G_{2}$ so that $\left(g_{1}, h_{\infty}\right)$ is a point of joint continuity for $C$. To conclude the proof, we shall show that all the points of $G_{1} \times G_{2}$ are points of joint continuity for $C$.

Indeed, let $\left\{g_{n}^{\prime}\right\}$ be a sequence from $G_{1}$ such that $\lim _{n \rightarrow \infty} g_{n}^{\prime}=O_{G_{1}}$, and let $\left\{h_{n}\right\}$ be a sequence from $G_{2}$ such that $\lim _{n \rightarrow \infty} h_{n}=h_{\infty}$. Since $\left(g_{1}, h_{\infty}\right)$ is a point of joint continuity for $C$, we have that $\lim _{n \rightarrow \infty} C\left(g_{1}+g_{n}^{\prime}, h_{n}\right)=C\left(g_{1}, h_{\infty}\right)$. Now, the biadditivity of $C$ implies that $C\left(g_{1}+g_{n}^{\prime}, h_{n}\right)=C\left(g_{1}, h_{n}\right)+C\left(g_{n}^{\prime}, h_{n}\right)$. Since $C\left(g_{1}, \cdot\right): G_{2} \rightarrow G_{3}$ is continuous, we see that

$$
\lim _{n \rightarrow \infty} C\left(g_{1}+g_{n}^{\prime}, h_{n}\right)=\lim _{n \rightarrow \infty} C\left(g_{1}, h_{n}\right) .
$$

Therefore $\lim _{n \rightarrow \infty} C\left(g_{n}^{\prime}, h_{n}\right)=O_{G_{3}}$, and clearly this last result remains valid for any other sequences such that $g_{n}^{1} \rightarrow O_{G_{1}}$ and $h_{n}^{1} \rightarrow h_{\infty}$. Now, let $\left\{g_{n}^{*}\right\}$ be a sequence from $G_{1}$ such that $\lim _{n \rightarrow \infty} g_{n}^{*}=g_{1}$ and let $\left\{h_{n}^{*}\right\}$ be a sequence from $G_{2}$ such that $\lim _{n \rightarrow \infty} h_{n}^{*}=O_{G_{2}}$. Then, a similar argument shows that $\lim _{n \rightarrow \infty} C\left(g_{n}^{*}, h_{n}^{*}\right)=O_{G_{3}}$. From which, using the biadditivity of $C$, we can deduce easily that $C$ is jointly continuous at every point of $G_{1} \times G_{2}$.

The second half of the proof of Theorem 1 together with [1, Corollary 4, p. 648] yield the following result.

THEOREM 2. Let $G_{1}, G_{2}$ and $G_{3}$ be abelian complete metrizable groups. Let $C: G_{1} \times G_{2} \rightarrow G_{3}$ be biadditive and separately continuous. Then $C$ is jointly continuous.

3. Christensen measurable mappings. First we prove the following lemma.

LEMMA 1. Let $G$ be an abelian Polish group. Let $S$ be a universally measurable subset of $G$ such that $G \backslash S=S^{c}$ is a Haar zero set. Let $u$ be a probability measure on $G$. Then there exists a $g \in G$ such that $u(S+g)=1$.

ProOF. Let $v$ be a probability 'testing' measure for $S^{c}$, i.e.

$$
\left(\chi_{S^{c}} * v\right)(x)=\int_{G} \chi_{S^{c}}(x+y) v(d y)=0
$$

Then by the Fubini theorem, we have that

$$
(u * v)\left(S^{c}\right)=\iint \chi_{S^{c}}(x+y) d u(x) d v(y)=\iint \chi_{S^{c}}(x+y) d v(y) d u(x)=0 .
$$

Hence $u\left(S^{c}+g\right)=0$ for $v$ a.e. $g$. Therefore for $v$ a.e. $g$ we have that $u(S+g)=1$.

Now, we can prove our main result. 
THEOREM 3. Let $G_{1}, G_{2}$ and $G_{3}$ be abelian Polish groups, let $C: G_{1} \times G_{2} \rightarrow G_{3}$ be a Christensen measurable biadditive mapping. Then $C$ is jointly continuous.

ProOF. As the first step, we show that there is a universally measurable set $S^{c} G_{1} \times G_{2}$ such that the restriction of $C$ to $S$ is universally measurable and $S^{c}=$ $\left(G_{1} \times G_{2}\right) \backslash S$ is a Haar null set. To see that, let $\left\{O_{n}\right\}$ be a countable base for the topology of $G_{3}$. Since $C^{-1}\left(O_{n}\right)$ is Christensen measurable, we have that $C^{-1}\left(O_{n}\right)=A_{n} \cup B_{n}$, where $A_{n}$ is universally measurable and $B_{n}$ is a Christensen zero set. Hence there exists an $N_{n}$ which is a Haar null set and so that $B_{n} \subset N_{n}$. Furthermore, $S_{n}=C^{-1}\left(O_{n}\right) \backslash N_{n}$ is universally measurable. Let $S=\bigcup_{n=1}^{\infty} S_{n}$, it is easy to show that $S$ has the required properties.

As the second step, we show that $C$ is separately continuous. To obtain a contradiction, suppose $g_{0} \in G_{1}$ is such that $C\left(g_{0}, \cdot\right): G_{2} \rightarrow G_{3}$ is discontinuous. Then there exist a sequence $\left\{h_{n}\right\}$ and $\varepsilon_{0}>0$ so that, $h_{n} \in G_{2}, d_{2}\left(O_{G_{2}}, h_{n}\right)<1 / 2^{n}$, and $d_{3}\left(O_{G_{3}}, C\left(g_{0}, h_{n}\right)\right) \geq \varepsilon_{0}$.

Let $H=\{0,1\}$ be the compact metrizable abelian group with Haar measure $\mu_{1}$ such that $\mu_{1}(\{0\})=\mu_{1}(\{1\})=\frac{1}{2}$. We define $\theta_{1}: H \rightarrow G_{1}$ by $\theta_{1}(x)=x g_{0}$. Let $K=\{0,1\}^{N}$ be the Cantor group with Haar measure $\mu_{2}$ which is the (countable) product of 'equidistribution' measures on $\{0,1\}$. We define $\theta_{2}: K \rightarrow G_{2}$ by $\theta_{2}(y)=$ $\sum_{n=1}^{\infty} y(n) h_{n}$ and $\theta: H \times K \rightarrow G_{1} \times G_{2}$ by $\theta(x, y)=\left(\theta_{1}(x), \theta_{2}(y)\right)$. Clearly, $\theta_{1}$, $\theta_{2}$ and $\theta$ are continuous mappings. By Lemma 1 we conclude that there exists a $\left(g_{1}, g_{2}\right) \in G_{1} \times G_{2}$ so that $\theta(H \times K)+\left(g_{1}, g_{2}\right)$ is essentially contained in $S$, in other words, $\theta^{-1}\left(S-\left(g_{1}, g_{2}\right)\right)$ has full Haar measure in $H \times K$. Therefore $D(x, y)=C\left(\theta(x, y)+\left(g_{1}, g_{2}\right)\right)$ is Haar measurable on $H \times K$, since it follows from our previous result that the composed map $D$ will be universally measurable on $H \times K$, except for a null set.

Let $\varepsilon$ be a fixed positive number. There exist balls $B_{0}$ and $B_{1}$ in $G_{3}$ of diameter less than $\varepsilon$ so that both of the sets $F_{0}=(\{0\} \times K) \cap D^{-1}\left(B_{0}\right)$ and $F_{1}=(\{1\} \times$ $K) \cap D^{-1}\left(B_{1}\right)$ have strictly positive Haar measure in $H \times K$. Now, by Pettis' theorem, we can conclude that there exists $N(\varepsilon)$ so that for $n \geq N(\varepsilon)$ we have that $\left(F_{0}+\left(\{0\} \times l_{n}\right)\right) \cap F_{0} \neq \varnothing$ and $\left(F_{1}+\left(\{0\} \times l_{n}\right)\right) \cap F_{1} \neq \varnothing$, where $l_{n}=$ $(0,0, \ldots, 0,1,0, \ldots)$.

Since $F_{0}=(\{0\} \times K) \cap\left\{(0, g): C\left(g_{1}, \theta_{2}(g)+g_{2}\right) \in B_{0}\right\}$ and $F_{1}=(\{1\} \times K) \cap$ $\left\{(1, g): C\left(g_{0}+g_{1}, \theta_{2}(g)+g_{2}\right) \in B_{1}\right\}$, it follows that for $n \geq N(\varepsilon) d_{3}\left(O_{G_{3}}, C\left(g_{1}, h_{n}\right)\right)$ $<\varepsilon$ and $d_{3}\left(O_{G_{3}}, C\left(g_{0}+g_{1}, h_{n}\right)\right)<\varepsilon$.

Now these inequalities imply that $d_{3}\left(O_{G_{3}}, C\left(g_{0}, h_{n}\right)\right)<2 \varepsilon$. Thus, by choosing $\varepsilon<\frac{1}{2} \varepsilon_{0}$, we got a contradiction, i.e. we have that $C$ is separately continuous.

Finally, we see that Theorem 1 yields the proof of this theorem.

\section{REFERENCES}

1. J. Calbrix and J. P. Troallic, Applications séparément continues, C. R. Acad. Sci. Paris 288 (1979), 647-648.

2. J. P. R. Christensen, On sets of Haar measure zero in abelian Polish groups, Israel J. Math. 13 (1972), 255-260.

3. _ Topology and Borel structure, North-Holland, Amsterdam, American Elsevier, New York, 1974.

4. J. P. R. Christensen and P. Fischer, Small sets and a class of general functional equations, Aequationes Math. (to appear). 
5. T. M. K. Davison, A functional equation for quadratic forms, Aequationes Math. 21 (1980), 1-7.

6. P. Fischer and Z. Slodkowski, Christensen zero sets and measurable convex functions, Proc. Amer. Math. Soc. 79 (1980), 449-453.

7. P. Fischer and J. P. Mokanski, A class of symmetric biadditive functionals, Aequationes Math. 23 (1981), 189-174.

8. A. M. Gleason, The definition of a quadratic form, Amer. Math. Monthly 73 (1966), 1049 1056.

9. S. Kurepa, On the quadratic functional, Acad. Serbe. Sci. Publ. Inst. Math. 13 (1959), 57-73.

10. P. Jordan and J. von Neumann, On inner products in linear metric spaces, Ann. of Math. (2) 36 (1935), 719-723.

MATEMATISK INSTITUT, KøBENHAVNS UNIVERSITET, UNIVERSITETSPARKEN 5, DK2100 KøBENHAVN $\varnothing$, DENMARK

DEPARTMENT OF MATHEMATICS AND Statistics, UNiVERSity OF GuelPh, GUELPH, ONTARIO, CANADA 